Check for updates

Montreal

Cite this as: BMJ 2021;375:n3013

http://dx.doi.org/10.1136/bmi.n3013

Published: 03 December 2021

\section{Covid-19: South Africa's surge in cases deepens alarm over omicron variant}

Owen Dyer

South Africa, the first country to detect the omicron variant of the SARS-CoV-2 virus, is seeing an unprecedented surge in cases, lending weight to concerns that this variant may prove to be the most transmissible yet seen.

The number of new cases among people who have had a previous positive test suggest that omicron is much more likely than previous variants of concern to reinfect people who have had covid, said the country's National Institute for Communicable Diseases.

In the outbreak's epicentre-Gauteng, the populous province that contains Pretoria and

Johannesburg $-74 \%$ of samples sequenced from the last three weeks of November involved the new variant. This suggests that omicron is rapidly outcompeting the prevalent delta variant, which had already replaced the local beta variant. With only scattered cases identified in at least 23 other countries, South Africa's experience currently offers the clearest glimpse of the variant's possible future course.

"Based on mathematical modelling conducted by ECDC [the European Centre for Disease Prevention and Control], there are indications that omicron could cause over half of all SARS-CoV-2 infections in the EU/EEA within the next few months," the ECDC said in a statement on 2 November. The French government's scientific adviser, Jean-Francois Delfraissy, said that omicron could take delta's place by the end of January.

The number of new daily cases in South Africa is doubling at a much faster rate than in any of the country's three previous waves. The seven day rolling average of new daily cases stood at 266 when the first sample was isolated on 8 November, at 674 when South African scientists identified it as a possible new variant of concern on 23 November, and at 5092 on 2 December.

The surge still appears to be accelerating, shown by the proportion of tests returning positive, which rose from $10.3 \%$ of tests ( 4373 cases) on 30 November to $16.5 \%$ of tests on 1 December (8561 new cases), $22.4 \%$ of tests on 2 December (11 535 new cases), and 24.3\% of tests on 3 December (16 055 new cases).

The rate of deaths has yet to show a similar rise. Anecdotal reports from South African hospitals suggest that young people feature prominently among new cases. "We've seen quite a sharp increase [in hospital admissions] across all age groups, particularly in the under 5s," said Wassila Jassat, an epidemiologist at the National Institute for Communicable Diseases.
South Africa has a low vaccination rate at about 36\% of the adult population, but the rate is particularly low in young people, especially men. "Fake news in the social media space has had a huge impact" among this demographic, said the health minister Joe Phaahla last month.

The first identified samples were collected on 8 November from a 34 year old man and a 23 year old man in Johannesburg.

\section{Reinfection risk}

There are still no data on the vaccination status of people infected with omicron. On the basis of its known mutations, vaccine makers have expressed optimism that immunisation will continue to protect against the worst outcomes of covid-19 brought on by South African researchers found that it could be more dangerous than other variants to people whose immunity was acquired through previous infection. ${ }^{1}$

The country has experienced three previous waves, driven by the original virus, the beta variant, and the delta variant. In the rapidly building omicron surge-yet to be officially declared a fourth wave-the proportion of new cases involving people who have already experienced covid-19 is more than three times higher than in the second and third waves, the study found.

If this finding is borne out elsewhere, omicron would pose a particular danger in countries with high rates of natural immunity but relatively low rates of vaccination, such as South Africa, Russia, and the United States.

\section{HIV connection suspected}

Tulio de Oliveira, the South African scientist who identified the omicron variant, told the Los Angeles Times that "the most plausible" story of the variant's origin was that its multiple mutations accumulated in the body of a person with HIV whose compromised immune system struggled to clear the virus for weeks or months. ${ }^{2}$ South Africa was previously the likely point of origin of the beta variant of concern.

As the country has an HIV prevalence of $19 \%$ in people aged 15-49, its large untreated population could "become a factory of variants for the whole world," warned de Oliveira. With Africa's high HIV prevalence and a continent-wide vaccination rate of under $7 \%$, it has been left behind in the pandemic, he and colleagues wrote in Nature. ${ }^{3}$

Travel bans have effectively punished South Africa for swiftly notifying the World Health Organization of the variant, they noted, which has discouraged transparency. The many flight cancellations are now by the new variant. But a preprint study posted online 
causing a shortage of reagents for test kits that South Africa needs to track the omicron surge, said the National Institute for Communicable Diseases, and WHO warned that they were also preventing the dispatch of samples abroad.

1 Pulliam JRC, van Schalkwyk C, Govender N, etal. Increased risk of SARS-CoV-2 reinfection associated with emergence of the Omicron variant in South Africa. 1 Dec 2021. [Preprint]. https://www.medrxiv.org/con-

tent/10.1101/2021.11.11.21266068v2.full.pdfdoi: 10.1101/2021.11.11.21266068

2 Healy M. Did failure to adequately treat HIV patients give rise to the Omicron variant? Los Angeles Times2021 Dec 2. https://www.latimes.com/science/story/2021-12-02/did-omicron-coronavirusvariant-arise-in-patient-with-uncontrolled-hiv

3 Msomi N, Lessells R, Mlisana K, et al. Africa: tackle HIV and COVID-19 together. Nature 2021 Dec 1. https://www.nature.com/articles/d41586-021-03546-8

This article is made freely available for use in accordance with BMJ's website terms and conditions for the duration of the covid-19 pandemic or until otherwise determined by BMJ. You may use, download and print the article for any lawful, non-commercial purpose (including text and data mining) provided that all copyright notices and trade marks are retained. 